\title{
Erhu Concerto Since the New Century
}

\author{
Jindi Zhang
}

Art College, Shandong University, Weihai, 264209, China

\section{Keywords: New century, Erhu concerto}

\begin{abstract}
Since the new century, erhu works compared to the 90 s had the very big change, in the 90 $\mathrm{s}$, the popular music creation mainstream position, in the 2000 years later, professional music creation back to the position of the mainstream. This development is affected by the age of economic and cultural development, but also by the $90 \mathrm{~s}$ and after the increase in the number of exchange interaction in Taiwan. This article will select three composers Wang Jianmin, Guan Naizhong, Gao Shaoqing was analyzed and reviewed in this paper.
\end{abstract}

\section{Erhu concerto artistic characteristics}

\section{Erhu playing skills and acoustic development}

In the new century, with the diversification of erhu technical control and more rich diversity, is to grasp the style of traditional techniques and skills to master modern style, technology across difficulties, strengthen the comprehensive technical control.

Guan NaiZhong have adopted some elements of traditional music in later writings. The commitments Beijing "works using the Beijing style drum, XiPi, against Er huang, monochord, in" resolutely encourage ", "love/hate", "mind autumn in wind and rain"used in the works is Guchin music material.

Several of its creation characteristics of all is the use of traditional music notes as a start, appeared immediately jump out to develop new idea, the beauty such as drums or Guchin music element and its lasting appeal into different style of dance music rhythm, building into a new style of traditional music. The performance of the works without using the techniques, very hard not to show off and specially arranged a skill, but according to the requirements of the works of music development, apply the techniques of certain difficulty in the layout of the entire piece of music. Work, for example, using large jump stereo music to increase the span of the feeling and the sense of speed, strong to auditory stimuli, to change the original single melody of erhu music hearing feeling, form unique sound effects. In proportion to the big jump techniques used in the traditional music is very small, but in modern music works, for use in the works of Guan NaiZhong more technique as its characteristics, and has the characteristics of Guchin shock characteristics, perhaps this is the result of his wife good play Guchin inspiration and reference for many of the harp music.

Wang Jian min's four rhapsody works material respectively based on music of ethnic minorities in Yunnan, Hunan folk songs and opera, the style of the western regions in Xinjiang, northwest folk tones. Its creation characteristic is made new again after folk tones for purification of artificial scales, combined with its unique core interval relations processing again into a new melody. Work performance skills in the use of in the end of the rhapsody presto tend to use difficult technique, plate part of speed up to 180 more rapidly, $\mathrm{J}=180$ quick change and big jump connect, fast hands with bow, fast, bow technique are extremely hard skills, etc. And by extending the interval distance, jumped into the continuous use intervals, the span of several appoggiatura reached 10, 12 degrees, and even once 15 degrees, and the development of the tone conversion technique to break the traditional technique, the technique of using fall of erhu technique difficulty highest use.

Gao Shaoqing's works of four caprices material based on the Mongolian music, Irish dance music, and pop music or create melody. The composing characteristics is that love with pentatonic scale as the main melody, rhythm feeling is stronger, faster and with grace note.

The three types of work for the use of rhythm is a prominent point. Guan NaiZhong works is to use a triplet, segmentation rhythm, dotted notes, semiquaver, bow to use weak up in the bow and the use of explosive force is the wind of Guchin; Wang Jianmin's works from the beat to the rhythm of the 
changes are heavy and complicated, with more frequent than the use of other composers; Gao Shaoqing's work rhythm in the rhythm of the popular style, such as jazz and artificially with accent marks, cause the feeling of misplaced accent. The composer's work on the rhythm used to break the routine use of erhu music rhythm, using a variety of irregular rhythms changed the original rhythm; strengthen the rhythm of auditory impact dynamic sound effects.

Works under the new creative ideas to show the new melody and rhythm of playing technique and the combination of various playing traditional music style technology of erhu playing technology is a big challenge. A lot of big jump use requires more adept and natural skills to show works of music expression, it needs to be in peacetime through a series of scales and arpeggios fast and big jump exercises to exercise finger flexibility, reaction ability and position, etc., to ensure the stability of the intonation. Need a series of all kinds of beats, rhythm type style rhythm exercises to exercise heart hand unified rhythm feeling, sense of rhythm. Work traditional music style technology assurance is a big difficulty, playing techniques that need to learn and master all kinds of folk music expression, coupled with new skill mix, become a new language, the characteristics of the style of expression, this kind of technique of playing skills to master to pay attention to the moderate play discretion, namely to achieve "spirit likeness" rather than "like", to grasp the "charm" of express, to express the real soul of music.

\section{Western composing techniques and blending of Chinese aesthetics}

The composer of the new century to his work after a period of time after the practice and test, got a confident after washing, in the music creation also will go to adjust and relatively rational thinking, pay attention to the creation of all aspects of the factors.

"Zhui Meng Jing Hua" is a title of work that made by Mr Guan NaiZhong in 2002 impression of childhood old Beijing reminiscing about. The two empty strings sound of erhu music adopted A, D began tuning as starting point, introducing music thoughts, don't have the idea. A beautiful melody comes from clever and creative, a good performance is the result of the tuning accurately, a year the four seasons, spring, summer, autumn, winter comes from germinating in spring, a busy life is the result of the cycle of life.

The author works on the commentary of the mind, spirit and tips on how to play the natural, no title work compared to the pure music to express ideas, more pay attention to the humanities culture and the combination of music, and the interaction between creators and performers and appreciators. Performers in the second creation of the perfect interpretation of the music, let the composer of erhu works with greater confidence and inspiration, in the same way, the composer of this piece of work has been more than a decade of silence and also a not to find a more suitable for China's national conditions in the reflection of music expression, composition of strategy adjustment, change the pure music is not to be understood from embarrassment.

\section{The artistic quality of works of form and content, literary characteristics}

a). The pursuit of personality, the communion between Chinese and western.

b). Compatibility of Chinese and western, natural and hair.

\section{The development of erhu concerto composing and performing art}

Since 2000, various contest held by catalysis on erhu works of creation, introduced many new erhu works, works of "the wind and rain, autumn", "be the wind", "desert", "the second, third, fourth rhapsody" and so on are the national erhu play final works specified. Stimulus in the game under the condition of work often have a match of the birth of the functional characteristics, performance techniques become creation must consider the contents of the difficulty, the frequent games held after 2000, stimulate the creator constantly improve the quality of his work and difficulty, especially Wang Jianmin's first "rhapsody" four works, each capital is entries, forcing the creator of his own work to do first and foremost a breakthrough, prompting works than poem difficult, technical difficulty and almost reached the limit of erhu music.

The individual character has highest performance for characteristic of works in this period. Analysis from the identity of the three composers experience, a long-term life abroad, a position 
between the Chinese and foreign life, a long-term domestic life, the creation idea, thinking, aesthetic and so on have very big difference.

Wang Jianmin's music creation is using theoretical analysis and ponders, after the examination of the creation of the music model. By means of modern western music creation, for example, on the pitch gene derived core tone (2, 4 degrees of northwest, exhibition into seven degrees, five degrees) based on creation, claims to write a, Volkswagen is fit with Chinese style, contemporary works, the most important thing is to promote the development of the discipline, to be nice don't stick. Erhu melody is always dominated, so pay attention to the melody writing, like positive to arouse the enthusiasm of the people. Who wants to write some suitable for China's national language and have their own style of work. And Gao Shaoqing under the practice of rich experience, with erhu in practice has already been applied to natural fusion of Chinese and western cultural elements, and has formed a cultural habits, using this cultural habits for music creation. This habit had a habit of cultural integration, also have skill fusion style habits. And Wang Jianmin is to analyze a material refining to reconstruct into a new language to compose, this is just a kind of writing style and idea of the creator, just consider the music from the Angle of creation, and psychological habit of playing but not the most direct experience, skills, habits, is taking to create an idea. The performer is often with a kind of love and desire to create, as Gao Shaoqing concept, technique is not the main, the key to good major. Guan NaiZhong works of creation, between the new language while absorbing the elements of modern music, but mostly is based on a Guchin music, formed on the techniques of the span of the language, although the instrument performance is different, culture is divided into low, but the cultural aesthetic is also a national culture, the works of traditional in the later work more.

Gao Shaoqing cannot think erhu with changeless should change, die practice skills basic skills, should be open, flexible language single way of music playing her own will feel boring, the audience will not be touched and loved nature. With the Angle to express the works of the players, the use of music language, playing techniques will there be new impromptu sexual performance.

\section{Representative works and characters}

Gao Shaoqing is a famous erhu musician, composer, the most outstanding, one of the most innovative erhu players.

Gao Shaoqing was born in 1979 and was admitted to Shanghai music institute accessorial middle school and university. In 1991 and was admitted to the royal Canadian college of music, learning piano and composition. In 1993, became the first professor in Toronto Royal Academy of music history teachers of Chinese national Musical Instruments, and formally set up erhu professional teaching course. Gao Shaoqing had transplanted and played the violin music "infinite move", "fantasia Carmen," song of a wanderer "and other classical violin. During the period of domestic organized and participated in the "red maple leaf" rock band and pop band "snowman". In 2006 by the 11 pair of stringed instruments "bow" flame band, the band fusion of classical, jazz, rock, world music elements, such as formation in the form of multiple music playing style. Gao Shaoqing in the orchestra as erhu hand, orchestra playing style for Gao Shaoqing of erhu and erhu creation has a huge influence.

He introduced the jazz, rock, South American music, pop music and country music elements into erhu music creation, the drovers' spirit, "three miles pave", "youth dance" and so on with the jazz style of Chinese folk music into the world trend of The Times. He to film, television, the musical score playing: "made in heaven", "Thai star guest"," the bird of a foreign land", etc.

People living vision to expand and its own aesthetic, the demand of the senses, would change with the progress of the social life and the pursuit of music and experience. Gao Shaoqing made the experience into the creation motive, flows naturally from the bottom of erhu art have a passion for music creation of erhu caprices series started in 2002, to 2012 decade created four works: "homesickness", "be the wind", "moves", "desert".

Capriccio is a kind of music genre, is a general term for music composer created by fantasy. Formal structure is free, rich life and randomness. The author chooses this subject is to hope to be able to free to express inner enthusiasm is bold and unrestrained feelings. 


\section{Erhu concerto teaching and theoretical research}

Since 2000, the emergence of a lot of new works to erhu art provides a display platform, but also give people ponder question. On the one hand, the development of erhu technology more mature is welcome, but in competition and frequent phenomenon is gone after blindly technique, the sorrow, as major part of the works of art is a reflection of art, the taste of this kind of art requires national culture infiltration to express it, this is the usual accumulate over a long period of contact to study these traditional music culture can have this kind of music quality. About the style of practice teaching materials in this period have Wang Guotong's "style of erhu etudes", triple of the erhu solo 20: hard rock, soft rock and roll style "and" Bruce ragtime style ", "jazz swing style", "country" the beetles style of music "series pop music style etudes, etc. On the one hand, the development of erhu music there is still a lack of theoretical summary and research work, including erhu teaching material of systematization, the standardization of the erhu education, construction of erhu art theory has yet to be speed up to keep up with the development of creation and playing erhu art. This period has outstanding contribution to the theory of erhu is Chen wei "erhu art history", carries on the comparison to Chinese erhu art system, the research work, and Qiao Jian-zhong, Yang Guangxiong editor of "the fallow ceremony, erhu volume".

After 2000 year, the universities have set up a music department, this music from outside of the professional art colleges and universities of another culture and enrich the development, the development of erhu art a multi-layer link supplementary development. But in these comprehensive universities music education is not suitable for this level of standard, unified teaching and education system, and it will be a need to further efforts to complete a career.

\section{Conclusion}

Since the period of the development characteristics in the new century, in general, large work piece length, melody structure using single movement, movement, body form. Series is one of the features of this period. Relative to the $90 \mathrm{~s}$, the creation is more inclined to create professional quality. Subject material more style characteristics, and using modern vocabularies fusion, as a result, technology of comprehensive make use of this period should not only pay attention to professional training style, and to pay attention to the training of new skills. In the early $90 \mathrm{~s}$ following a diversified development in the $90 \mathrm{~s}$, relative to the professional nature, middle and late development into a pandemic, universal characteristic.

\section{References}

[1] Liu Changfu. The erhu comprehensive performance skills, Musical Instruments, 2004(10), 34. 